\title{
A Time-Response Model for Analysis of Drug Transport and Blood Flow Response during Iontophoresis of Acetylcholine and Sodium Nitroprusside
}

\author{
Erik Tesselaar $^{\mathrm{a}}$ Joakim Henricson $^{\mathrm{b}}$ Susanne Jonsson ${ }^{\mathrm{a}} \quad$ Folke Sjöberg $^{\mathrm{b}, \mathrm{c}}$ \\ Departments of a Medicine and Care, ${ }^{\mathrm{b}}$ Biomedicine and Surgery, and ${ }^{\mathrm{C}} \mathrm{Hand}$ and Plastic Surgery and Intensive Care, \\ Faculty of Health Sciences, Linköping University Hospital, Linköping, Sweden
}

\section{Key Words}

Time-response model $\cdot$ Iontophoresis $\cdot$ Laser Doppler

flowmetry $\cdot$ Acetylcholine $\cdot$ Sodium nitroprusside

\begin{abstract}
Background/Aims: The analysis of blood flow responses to iontophoresis of vasoactive drugs is often limited to evaluation of maximum responses. In this study, a time-response model is proposed for the blood flow responses to vasoactive drugs applied by iontophoresis. Methods: The microvascular bed is represented as a single compartment with a zero-order influx of the drugs from the electrode and a firstorder clearance due to diffusion and blood flow. The blood flow response to the local drug dose is described using the $\mathrm{E}_{\max }$ model. Results: The model accurately describes the blood flow responses to acetylcholine and sodium nitroprusside during a single iontophoretic current pulse. There is a significant clearance out of the microvascular bed during iontophoresis which depends on the type of drug administered. Conclusion: The model enables an accurate estimation of response parameters such as ED50 and maximum response, even if the true maximum blood flow is not obtained. The results suggest that due to clearance from the microvascular bed, the local drug dose during a single pulse of current is not linearly proportional to current strength multiplied by pulse duration.

Copyright $\odot 2008$ S. Karger AG, Basel
\end{abstract}

\section{Introduction}

Transdermal iontophoresis is a method of drug delivery through the skin by application of a small electric current. When vasoactive drugs are delivered by iontophoresis, the reactivity of the microvasculature to these drugs can be assessed using noninvasive techniques such as laser Doppler flowmetry [1-3]. Iontophoresis of vasoactive drugs has a number of advantages compared with other methods. It provides a convenient, noninvasive method and does not induce systemic drug effects. A limitation of iontophoresis, however, is the fact that the local drug dose in the skin is unknown. This limitation has been dealt with in general by using constant-current iontophoresis and assuming that the drug dose in the skin is proportional to the applied iontophoretic charge, which is defined as the current strength multiplied by the duration of current application [4].

In the many studies using iontophoresis and laser Doppler flowmetry, the parameter of interest has been the maximum blood flow response, defined as either an absolute value or a relative increase $[5,6]$ or as an area under curve $[7,8]$ after a certain period of iontophoretic current application. However, this approach has several limitations. First, the maximum response seen during an experiment may not reflect the true physiological maximum. Especially when plateaus in the responses are ab-

\section{KARGER}

Fax +4161306 1234 E-Mail karger@karger.ch www.karger.com
Dr. Erik Tesselaar

Department of Medicine and Care

Linköping University Hospital

SE-581 85 Linköping (Sweden)

Tel. +461322 18 20, Fax +46132228 36, E-Mail e.j.tesselaar@gmail.com 
sent, the accuracy with which the maximum physiologic response can be measured may not be very high. Second, the response over time to a drug that is applied by iontophoresis contains more information than the maximum response alone. By considering only the maximum response, important physiological characteristics may be overlooked. For example, in a previous study we found that the maximum response in healthy volunteers to acetylcholine (ACh) was not different from the maximum response to sodium nitroprusside (SNP). However, the dynamics of the responses were very different. By fitting a sigmoidal curve, commonly used in pharmacological in vitro studies, to the responses, we could quantify these differences in terms of ED50 and Hill slope parameters [3].

The advantage of fitting such a sigmoidal curve to the blood flow response data is that it yields dose-response parameters that can be easily quantified and compared between drugs or patient groups. Still, the limitation of fitting the vascular response with an empirical model is that it is not based on the physical processes underlying the response to the drug in question [9].

In pilot experiments, we have noticed that when different combinations of current strengths and pulse durations were used while iontophoretic charges were kept equal (that is, constant charge of $12 \mathrm{mC}$ using $0.02 \mathrm{~mA} \times$ $600 \mathrm{~s}, 0.04 \mathrm{~mA} \times 300 \mathrm{~s}$ and $0.06 \mathrm{~mA} \times 200 \mathrm{~s}$ ), the response curves were shaped differently. Maximum responses as well as ED50 values differed for the different current strengths used. This led us to believe that the commonly used assumption that dose is proportional to current strength multiplied by pulse duration may not be valid.

The primary aim of this study was to develop a mechanistic time-response model, in which both the physical transport of drugs into the skin and the physiological response to these drugs are taken into account, and to apply this model to the blood flow response to ACh and SNP. The secondary aim was to investigate whether this model is able to explain the finding that the blood flow response to equal iontophoretic charges differs for different combinations of current strength and time.

\section{Theory}

\section{Time-Response Model: Drug Kinetics}

When a vasoactive drug is transported into the skin by iontophoresis, its pharmacological action occurs primarily within the microvascular bed, which is embedded in the subdermal plexus. To model the drug dose, $D$, in this cutaneous microvascular bed, a single-compartment model is proposed in which the drug is transported according to a mass transfer process.

During iontophoresis, drug molecules can be transported into the skin by passive diffusion, electrorepulsive forces and electroosmosis. For the iontophoretic transport of small ionic drugs, the major transport mechanism is the electrorepulsive force [10]. Calculation of the amount of drugs transported due to iontophoretic charge shows that this amount is typically less than $1 \%$ of the total drugs in the electrode. It is therefore unlikely that during the measurement, the transport is limited by a decreasing amount of drug or by inhomogeneities in drug concentration within the electrode, even if some degradation of the drug occurs. Hence, we assume that, at least for small ionic drugs, the influx of drugs is constant and that no depletion of drugs from the iontophoresis electrode occurs (the mechanism of transport for uncharged drugs is different and the influence of pore size, pore charge and background electrolytes becomes important).

The flux of drug ions from the iontophoresis chamber into the skin compartment, $J$, can then be described as a zero-order process, which is proportional to the current strength used:

$$
J=\frac{n}{Z F} I
$$

where $I$ is the electrical current strength in Coulombs/ second, $n$ the transport number, that is, the fraction of transported ions which are drug ions, $Z$ the valence of the drug ions and $F$ Faraday's constant.

In the skin compartment, the drug ions are removed by at least 2 mechanisms. Drug ions are assumed to move from the microvascular bed to surrounding skin layers by passive diffusion. Also, blood flow actively transports the drug ions out of the skin compartment. This removal by blood flow is not easily described, as the blood flow itself is a nonlinear function of the drug concentration in the case of vasoactive drugs. As a first approximation, we propose a first-order kinetic process with a rate constant, $\lambda$, for the combined passive and flow-mediated removal of drugs from the skin compartment.

These mechanisms of transport of drug ions in and out of the skin compartment can be described by the following ordinary differential equation:

$$
\frac{d D(t)}{d t}=J-\lambda D\left(t-t_{0}\right)
$$


A certain time is required for the drug ions to move from the iontophoresis chamber to the skin compartment in which they act on the microvessels. Therefore, a lag time parameter, $t_{0}$, is incorporated in the model. No drug ions are present in the skin at time $t \leq t_{0}$, so $D\left(t_{0}\right)=0$. By solving equation 2 using this initial condition, the drug dose in the skin compartment as a function of time can be found:

$$
D(t)=\frac{n I}{Z F \lambda}\left(1-e^{-\lambda\left(t-t_{0}\right)}\right)
$$

When the clearance rate constant, $\lambda$, and the lag time, $t_{0}$, are both reduced to zero, the model reduces to a form that represents the conventional assumption that the dose is proportional to the current strength multiplied by the pulse duration:

$$
D(t)=\frac{n}{Z F} I t \quad\left(\text { for } \lambda=0 \text { and } t_{0}=0\right)
$$

\section{Time-Response Model: Response Dynamics}

To describe the microvascular response to the drug ions in the skin compartment we use the $\mathrm{E}_{\max }$ model [11]. This model assumes that an agonist drug binds to receptors according to the law of mass action, and that the effect that the agonist generates is proportional to fraction of occupied receptors. This model can be described by one of the forms of the Hill equation:

$$
E(D)=\frac{E_{\max } D(t)}{D(t)+K}
$$

where $E_{\max }$ is the maximum possible response to the drug and $K$ is the drug dose that elicits half the maximum response. Substituting equation 3 in equation 5 gives the blood flow response as a function of time:

$$
E(t)=\frac{E_{\max }\left(1-e^{-\lambda\left(t-t_{0}\right)}\right)}{\left(1-e^{-\lambda\left(t-t_{0}\right)}\right)+\frac{\tau \lambda}{I}}
$$

where $\tau=K F Z / n$ is substituted as a parameter that combines the efficacy of the drug and the transport number and $E_{\max }$ is the maximum possible blood flow response. The model contains 4 unknown parameters: $E_{\max }, \lambda, t_{0}$ and $\tau$.

\section{Methods}

The models were applied to the blood flow responses to ACh and SNP, administered by a single-pulse, constant-current iontophoresis protocol, using 3 different current strengths.
Subjects

Ten healthy volunteers ( 5 men) with a mean age of 24.5 years (range 20-27) gave their informed and written consent before enrolling in the study. Reasons for exclusion were cardiovascular disease, diabetes, skin diseases and intake of medications (oral contraceptives excluded). Subjects refrained from using nicotine products and from ingesting alcohol or caffeine-containing beverages for $2 \mathrm{~h}$ before the experiments. During the measurements they were resting comfortably in a supine position. The study was approved by the medical ethics committee at the Faculty of Health Sciences, Linköping University, Sweden.

\section{Equipment}

Iontophoresis electrode chambers (Perimed AB, Stockholm, Sweden) were used for simultaneous application of drugs by iontophoresis and measurement of spatial and temporal blood flow variations by laser Doppler imaging. The inner diameter of the ring-shaped chamber was $14 \mathrm{~mm}$ (area $1.54 \mathrm{~cm}^{2}$ ), and the distance between the silver-silver chloride electrode ring and the skin was $2 \mathrm{~mm}$. A battery-powered iontophoresis controller (PeriIont 382; Perimed $\mathrm{AB}$ ) was used to deliver a constant electric current to the skin. A laser Doppler perfusion imager (PIM 2.0; Perimed AB) was used to measure skin perfusion. The details of the laser Doppler perfusion imager technique have been described earlier [3]. The perfusion was continuously measured at 16 distinct sites within the area of the electrode chamber and mean perfusion was calculated by the software at an interval of approximately $1 \mathrm{~s}$.

Drugs

Drug solutions were freshly prepared on the day of the measurements. Stock solutions of ACh (Miochol; Novartis AB, Stockholm, Sweden) and SNP (Nitropress; Abbott Laboratories, Abbott Park, Ill., USA) were dissolved in a mixture of deionized water and saline (Fresenius Kabi Norway AS, Oslo, Norway) to a final drug concentration of $1 \%$ and an ionic strength of $0.154 \mathrm{M}$.

\section{Iontophoresis Protocol}

The experiments were performed in a temperature-controlled room $\left(22.5-23.5^{\circ} \mathrm{C}\right)$. Three sessions were performed, in which the subjects received the treatments. The time between the sessions was at least $24 \mathrm{~h}$.

Each session was preceded by an acclimatization period of 10 min. During this time, the volar side of the subjects' forearm was gently cleaned with $70 \%$ ethanol and the electrode chambers were attached to the skin by double-adhesive tape. The electrode chambers were covered by a transparent plastic lid to avoid leakage and evaporation. Two different measurement sites, 1 for each drug, were used on the volar side of the forearm and a reference electrode was attached to the wrist. Positions on the forearm were chosen in order to avoid large vessels and damaged skin and the same positions were used throughout the sessions to reduce intrasubject variability.

Anodal iontophoresis was used to deliver ACh, cathodal iontophoresis was used for SNP. Both drugs were given by a current of $0.02 \mathrm{~mA}$ for $600 \mathrm{~s}$ [3], $0.04 \mathrm{~mA}$ for $300 \mathrm{~s}$ and $0.06 \mathrm{~mA}$ for 200 $\mathrm{s}$, depending on the session and in randomized order (each corresponding to a charge of $12 \mathrm{mC}$ ). In previous studies, we have found that such a charge does not elicit nonspecific responses [3, 


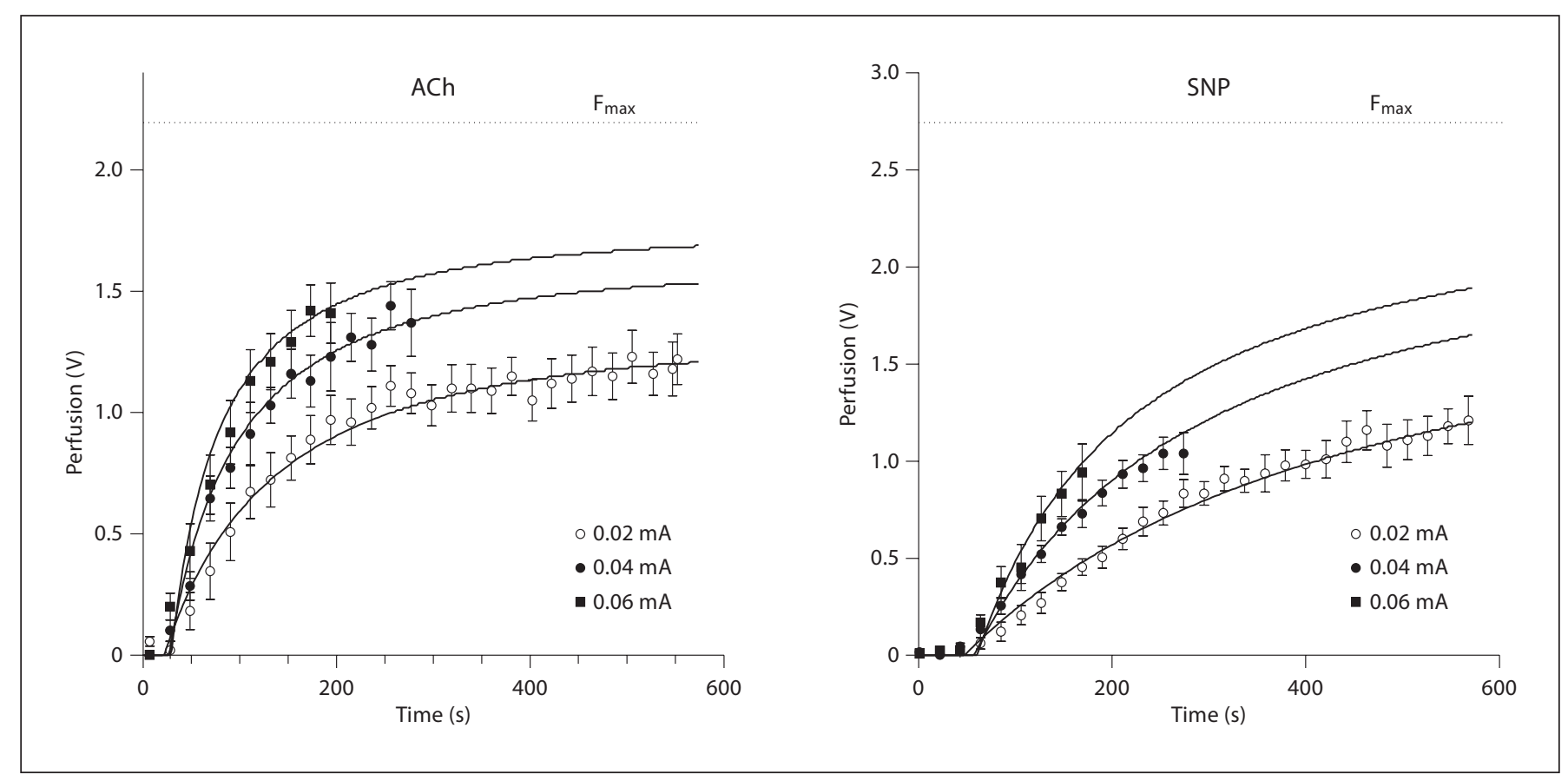

Fig. 1. Average blood flow responses to iontophoresis of ACh and SNP at different current strengths $(n=10)$. Solid lines are the responses predicted by the proposed time-response model.

12]. Before iontophoresis, the subjects' perfusion was recorded for $60 \mathrm{~s}$ to obtain baseline values. The average baseline perfusion during this period was subtracted from the blood flow data during iontophoresis. Drug delivery chambers were disposed after use.

\section{Data Analysis}

A nonlinear fitting procedure, based on the Levenberg-Marquardt algorithm, was used to fit the individual perfusion values from the laser Doppler imager to the time-response model. Before fitting the data, average baseline blood flow was subtracted from the perfusion values. Measurements with different current strengths $(0.02,0.04$ and $0.06 \mathrm{~mA})$ were performed in each subject. For each subject, the model parameters were obtained by global modeling. The parameters $E_{\max }, \lambda$ and $\tau$ were shared between the data at different current strengths, whereas $t_{0}$ was allowed to vary between different current strengths. The parameter $I$ was adjusted according to the current strength used. Average responses were calculated by taking the mean of the measured individual perfusion values at each time point.

The quality of the fitting was evaluated using the goodness-offit parameter $\mathrm{R}^{2}$. The goodness of fit of the time-response model was compared with that of a general logistic dose-response model, in which dose was defined as iontophoretic charge (current strength $\times$ pulse duration). Because the latter model is a reduced form of the time-response model, with the clearance rate $\lambda=0$ and the lag time $t_{0}=0$, an extra-sum-of-squares $\mathrm{F}$ test was used for this comparison [13].

Time-Response Model for Iontophoresis of ACh and SNP
When comparisons between model parameters were necessary, the significance of the difference of the mean values was tested using a paired two-tailed Student's t test. For all analyses, $\mathrm{p}<0.05$ was considered significant. Analyses were performed using GraphPad Prism version 4.00 for Windows (GraphPad Software, San Diego, Calif., USA).

\section{Results}

For both ACh and SNP, the speed of the response as well as the maximum blood flow measured depended on the current strength, with the highest current strength resulting in the fastest response and the highest maximum flow (fig. 1). The suggested mechanistic model could be fitted accurately to the blood flow data $\left(\mathrm{R}^{2}>0.96\right)$.

When the blood flow responses were described by a general signoidal dose-response model, in which dose was defined as current strength multiplied by pulse duration, the curves were right shifted for higher current strengths, making the overall curve fit less accurate (fig. 2). An extra-sum-of-squares $\mathrm{F}$ test revealed that for both drugs the proposed time-response model described the data significantly better than the conventional model in which $\lambda$ and $t_{0}$ are negligible ( $\left.\mathrm{p}<0.0001\right)$. 


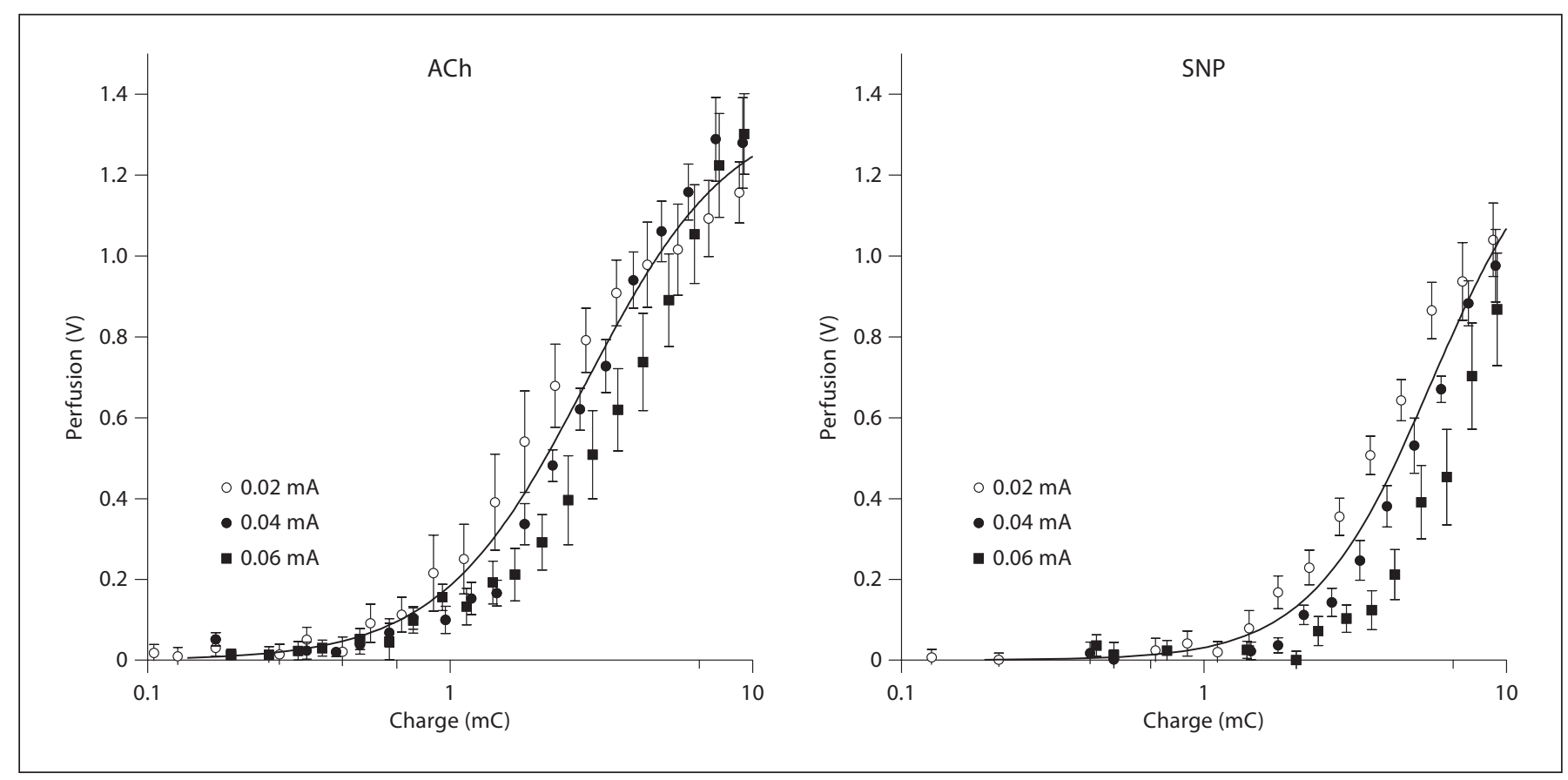

Fig. 2. Average blood flow responses to ACh and SNP, modeled using a sigmoidal dose-response model. Response curves obtained using different current strengths are horizontally shifted, indicating that charge and dose are not linearly proportional.

The clearance rate constant $\lambda$ for SNP was significantly smaller than for ACh. The lag time $t_{0}$ increased with increasing current strength and was significantly larger for SNP than for ACh. The parameter $\tau$, reflecting the effects of drug efficacy and transport number, was significantly larger for SNP compared with ACh. The maximum response parameter, $E_{\text {max }}$, was significantly larger for SNP. Parameters were estimated with narrow confidence intervals and are summarized in table 1.

\section{Discussion}

In studies of microvascular function using iontophoresis of vasoactive drugs, the amount of drugs in the skin as a function of time is unknown. A common assumption is that the administered dose is linearly proportional to the current strength multiplied by the pulse duration. The rationale for this assumption is Coulomb's Law, stating that a charge (carried by drug ions) is equal to the strength of the applied electric current (ions/second) multiplied by the time during which these ions are transported. Also, this relation has been verified in in vitro studies [4].
There are several drawbacks to this assumption. In practice, the current is not solely carried by drug ions. Background ions in the solution and counterions traveling from the skin towards the electrode compete with the drug ions, and the fraction of the current that is carried by drug ions is usually unknown [14]. Also, an iontophoretic dose is usually given over a certain period of time, typically seconds to minutes. During this time, in addition to the constant iontophoretic influx of drug ions, a clearance of drug ions from the skin due to passive diffusion and washout by blood flow may be expected [15]. To realize an instantaneous iontophoretic bolus, the current strength would have to be infinitely high, or at least much higher than the rate at which the drugs are removed from the skin. The maximum current strength that can be applied is in practice limited by the physiological tolerance to electric current.

In experiments where applied drug doses are unknown, time-response data often contain useful information about drug kinetics as well as pharmacodynamic characteristics [16]. In iontophoresis studies with vasoactive drugs, many investigators have described vascular responses in terms of maximum flow or area under curve [5-8]. However, the dynamics of the blood flow respons- 


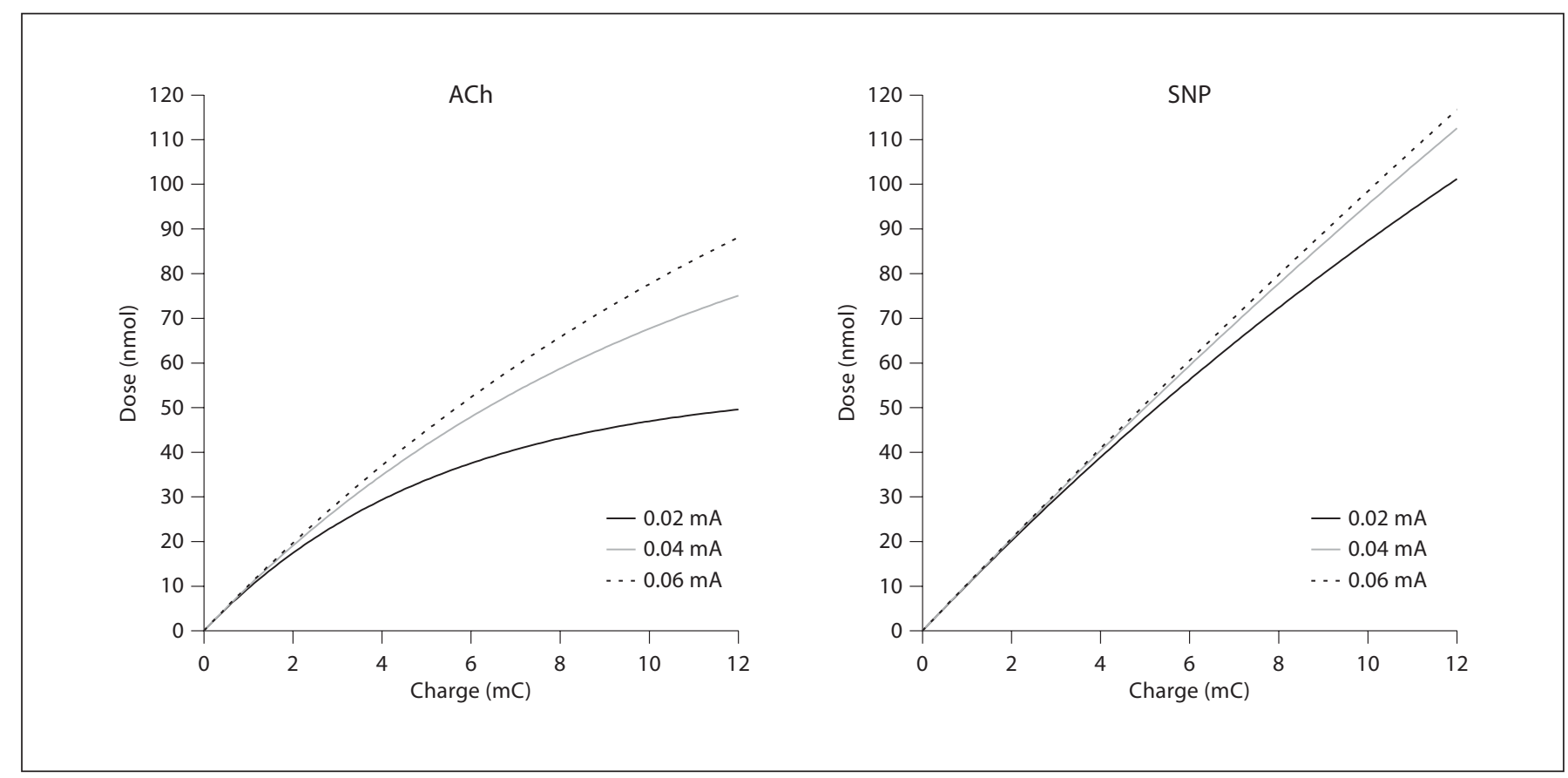

Fig. 3. Predicted doses delivered to the skin during iontophoresis of ACh and SNP, plotted as a function of iontophorestic charge and for different (constant) current strengths (assuming $n=1$ and $t_{0}=0$ ). The drug dose delivered to the skin is not linearly proportional with charge, especially with ACh and at lower current strengths.

Table 1. Best-fit parameters and 95\% confidence intervals of average blood flow responses to iontophoresis of ACh and SNP, obtained with the proposed time-response model

\begin{tabular}{|c|c|c|c|c|c|c|c|}
\hline \multirow[b]{2}{*}{$I, \mathrm{~mA}$} & \multicolumn{3}{|l|}{$\mathrm{ACh}$} & \multicolumn{3}{|l|}{ SNP } & \multirow[t]{2}{*}{$\mathrm{p}$} \\
\hline & 0.02 & 0.04 & 0.06 & 0.02 & 0.04 & 0.06 & \\
\hline$t_{0}, \mathrm{~s}$ & $21.8(19.6-24.1)$ & $27.0(26.0-27.9)$ & $29.2(28.5-29.9)$ & $47.3(44.2-50.4)$ & $57.4(56.4-58.4)$ & $60.5(59.5-61.5)$ & * \\
\hline$F_{\max }$ & & $2.1(2.0-2.2)$ & & & $2.7(2.4-3.0)$ & & * \\
\hline$l, \mathrm{~h}^{-1}$ & & $13.7(12.6-14.8)$ & & & $2.7(1.8-3.6)$ & & * \\
\hline$t, \mathrm{mC}$ & & $3.4(3.1-3.7)$ & & & $10.7(9.1-12.3)$ & & * \\
\hline
\end{tabular}

Figures in parentheses are $95 \%$ confidence intervals. The clearance rate $l$, efficacy-transport parameter $t$ and maximum response $E_{\max }$ are global parameters which are independent of the current strength. The lag time $t_{0}$ was allowed to vary between current strengths. * Significant difference between drugs.

es to different drugs or between patient groups may be fundamentally different, while maximum responses or areas under curve may be alike [3]. Expressing these differences in terms of differences in model parameters enables a quantitative comparison of results, rather than a descriptive conclusion that response curves are differently shaped. Moreover, applying a time-response model may lead to a better understanding of the underlying drug kinetics and pharmacodynamic actions.

In this study we have, for the first time, developed a model for iontophoresis of ACh and SNP which takes into account (1) the constant influx of drug ions by the electric current, (2) the clearance of drugs from the skin by passive diffusion and blood flow, and (3) the relation be- 
tween the local drug dose and the blood flow response based on common pharmacodynamic principles. The results of applying the proposed model to blood flow responses to ACh and SNP show that the model describes these responses accurately. This is confirmed by the narrow confidence intervals for the fit estimates.

When the responses were analyzed using conventional dose (charge) response curves, the curves for different current strengths did not overlap, but were right shifted for higher current strengths. Also, maximum responses were higher for higher current strengths. This indicates that the actual dose may not be linearly proportional to current strength multiplied by pulse duration, an assumption that is commonly made.

Our model was able to explain this finding. The clearance rate parameter $\lambda$ was significantly different from zero for ACh and SNP (13.7 $\mathrm{h}^{-1}$ and $2.7 \mathrm{~h}^{-1}$, respectively), and the model shows that the true physiological maximum response, $E_{\max }$, is never reached during the experiments because the dose in the skin is limited by the clearance rate and depends on the current strength. This effect is visualized in figure 3, where the estimated dose of drugs, as predicted by the time-response model, is plotted as a function of the iontophoretic charge and for different current strengths, assuming that all the current is carried by drug ions $(n=1)$ and with lag time $t_{0}$ neglected. As can be seen from the figure, the linearity between drug dose and charge increases with increasing current strength, but a high current strength is needed to obtain a linear relationship, especially for ACh.

Clearance of ACh from the skin was faster than clearance of SNP. This may have several causes. Different pharmacological mechanisms of ACh and SNP may result in different clearance rates. ACh must cross the vessel wall before it acts on muscarinic receptors on the intravascular endothelium and may be more susceptible to washout by blood flow in the vessels. On the contrary, SNP is known as a rapid-acting NO donor with a fast biotransformation. The NO molecule is released very easily from the nitroprusside and has a high affinity for its enzyme receptors. These receptors are located on the smooth muscle cells at the perimeter of the arterioles.

The parameter $\tau$ reflects both the efficacy of the drug (where a high value indicates a low efficacy) as well as the effect of the transport number. Although it is not possible to distinguish between the efficacy of the drug and the transport number in the current model, it is plausible that the transport numbers differ between ACh and SNP, since the drugs have opposite polarities. Transport of negative drugs such as SNP by iontophoresis is known to be more difficult than that of positive ions, since it is impeded by an electroosmotic solvent flow from the skin towards the cathode [10]. Therefore, slower transport of drugs into the skin may explain the larger value of $\tau$ for SNP and at the same time the larger estimate for the lag time $t_{0}$ that was found for SNP.

An unexpected finding in this study was that the lag time $t_{0}$ increased with increasing current strength. At higher current strengths, we expected the onset of the response to the drugs to occur earlier, as a result of an increased driving force or higher ionic mobility. Closer inspection of the onset of the blood flow responses reveals that there is no sudden onset at $t_{0}$ as assumed by the model, but that the response starts gradually and indeed earlier at higher current strengths. This unexpected finding can therefore be explained by the fact that the lag time provides a simplified representation of the dynamics at the onset of the response.

Although it seems that the dynamics of drug transport into and out of the skin cause the observed differences in maximum responses between current strengths, other explanations are possible. Blood flow during iontophoresis is known to increase as a result of current alone, an effect that is stronger with increasing current strengths. We think that it is unlikely that this nonspecific vasodilatation caused the differences in maximum responses between current strength for 2 reasons. First, the iontophoresis protocols we used in this study have been optimized in previous investigations, in which we found that they do not cause any nonspecific effects [12]. Second, the effect of nonspecific vasodilatation is known to be much stronger during cathodal iontophoresis. If a nonspecific effect would occur, it would most likely be seen in the responses to SNP. However, the responses with different current strengths were similar for ACh and SNP.

An important additional advantage of the proposed time-response model is that it is not absolutely necessary that plateaus in the responses are reached. Even though plateaus were not always reached in our experiments, especially in the case of SNP, fitting the model to the data resulted in accurate estimates of the maximum responses. However, this advantage comes at a cost, since measurements at different current strengths are required. It is not possible to accurately estimate all model parameters from measurements at a single current strength, due to the number of unknowns in relation to their effect on the shape of a single time-response curve.

The choice for a single elimination constant is an estimation. In reality, different elimination mechanisms are likely to be present. However, introducing a more com- 
plex parameter for elimination, or adding more parameters for different mechanisms, will make it considerably more complex to reliably estimate best-fit parameters. This is because the model will either become nonlinear or contain too many unknown parameters. We believe that a first-order elimination constant provides a reasonable approximation representing different elimination mechanisms, especially as long as the elimination rate is substantially smaller than the influx of drugs. This is the case in our experiments.

In conclusion, in this study we present a time-response model to describe the blood flow response to ACh and SNP applied by iontophoresis. The model takes into account both the transport dynamics of the drugs in the skin as well as the physiological response to the drugs. The results of fitting the model to the blood flow data indicate (1) that the model accurately describes the blood flow response to both ACh and SNP, resulting in well-defined estimates of lag time, drug clearance and maximum response, and (2) that there is a significant clear- ance out of the skin during iontophoresis, which is in conflict with the commonly used assumption that the administered dose during iontophoresis is linearly proportional to the current strength multiplied by the pulse duration. This finding has significant implications for the interpretation of results from studies on the microvascular response to vasoactive drugs applied by iontophoresis. Clearance of drugs from the skin limits the dose when low current strengths are used during longer periods, in particular with administration of ACh. It can be seen in figure 3 that the total administered dose of ACh using a current strength of $0.02 \mathrm{~mA}$ is about half the dose when $0.06 \mathrm{~mA}$ is used, even though the iontophoretic charges are equal. This may very well lead to false conclusions when studies are compared based on similar iontophoretic charges. The results also imply that to maximize the administered drug dose, short protocols using higher current strengths are preferable. However, total iontophoretic charge should be limited in order to avoid nonspecific responses.

\section{References}

1 Lindblad LE, Ekenvall L: $\alpha$-Adrenoceptors in the vessels of human finger skin. Acta Physiol Scand 1986;128:219-222.

$\checkmark 2$ Morris SJ, Shore AC: Skin blood flow responses to the iontophoresis of acetylcholine and sodium nitroprusside in man: possible mechanisms. J Physiol 1996;496:531-542.

-3 Henricson J, Tesselaar E, Persson K, Nilsson G, Sjoberg F: Assessment of microvascular function by study of the dose-response effects of iontophoretically applied drugs (acetylcholine and sodium nitroprusside): methods and comparison with in vitro studies. Microvasc Res 2007;73:143-149.

4 Phipps JB, Padmanabhan RV, Lattin GA: Iontophoretic delivery of model inorganic and drug ions. J Pharm Sci 1989;78:365369.

$\checkmark 5$ Abou-Elenin K, Xydakis A, Hamdy O, Economides PA, Horton ES, Veves A: The effects of aspirin and various iontophoresis solution vehicles on skin microvascular reactivity. Microvasc Res 2002;63:91-95.

6 Newton DJ, Khan F, Belch JJ: Assessment of microvascular endothelial function in human skin. Clin Sci 2001;101:567-572.
7 Åsberg A, Berg KJ, Hartmann A: Each administration of cyclosporin A enhances skin microvascular reactivity in renal transplant recipients. Microvasc Res 2000;60:81-90.

$\checkmark 8$ Anderson ME, Moore TL, Lunt M, Herrick AL: Digital iontophoresis of vasoactive substances as measured by laser Doppler imaging - a non-invasive technique by which to measure microvascular dysfunction in Raynaud's phenomenon. Rheumatology (Oxford) 2004;43:986-991.

9 Meibohm B, Derendorf H: Basic concepts of pharmacokinetic/pharmacodynamic (PK/ PD) modelling. Int J Clin Pharmacol Ther 1997;35:401-413.

$>10$ Singh J, Maibach HI: Topical iontophoretic drug delivery in vivo: historical development, devices and future perspectives. Dermatology 1993;187:235-238.

$>11$ Wagner JG: Kinetics of pharmacologic response. I. Proposed relationships between response and drug concentration in the intact animal and man. J Theor Biol 1968;20: 173-201.
12 Droog E, Henricson J, Nilsson GE, Sjoberg F: A protocol for iontophoresis of acetylcholine and sodium nitroprusside that minimises non-specific vasodilatory effects. Microvasc Res 2004;67:197-202.

13 Gabrielsson J, Weiner D: Pharmacokinetic and Pharmacodynamic Data Analysis: Concepts and Applications, ed 3. Stockholm, Swedish Pharmaceutical Press, 2002.

14 Mudry B, Guy RH, Begona Delgado-Charro M: Prediction of iontophoretic transport across the skin. J Control Release 2006;111: 362-367.

15 Nugroho AK, Della-Pasqua O, Danhof M, Bouwstra JA: Compartmental modeling of transdermal iontophoretic transport II: In vivo model derivation and application. Pharm Res 2005;22:335-346.

16 Gabrielsson J, Jusko WJ, Alari L: Modeling of dose-response-time data: four examples of estimating the turnover parameters and generating kinetic functions from response profiles. Biopharm Drug Dispos 2000;21:4152. 